\section{Hämangiome in den ersten Lebensmonaten therapieren}

— Klassische, lokalisierte Hämangiome sollten möglichst früh behandelt werden. Der ideale Zeitpunkt für den Eingriff ist die Proliferationsphase innerhalb der ersten vier bis acht Wochen. Dr. Heike Bause aus Michelstadt nannte als Therapieindikationen: Lokalisation in kritischen Bereichen wie Gesicht oder Anogenitalregion, Befall des behaarten Kopfes und von Fingern bzw.
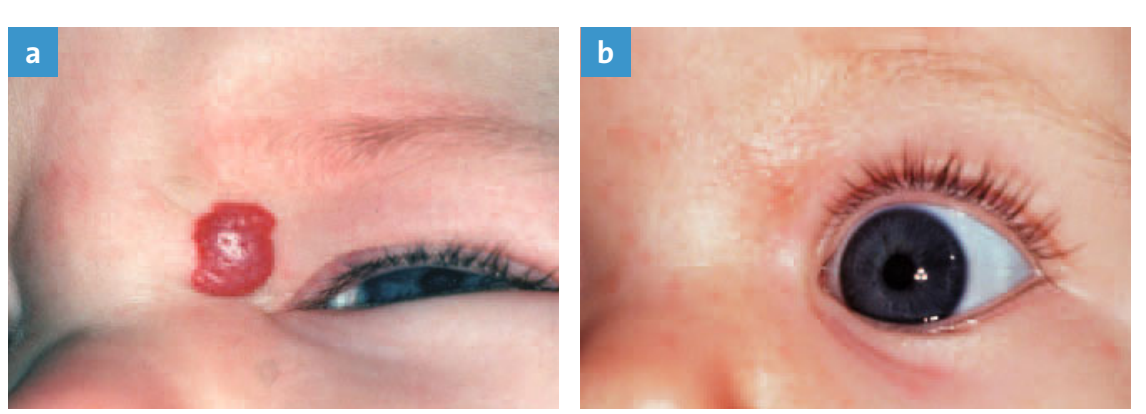

Kind mit Hämangiom: a: vor der Kryotherapie; b: Zustand nach der durchgeführten Kryotherapie

\section{Vitiligo im Blickpunkt}

— Eine unmittelbar nach der Geburt auftretende Vitiligo ist eine Rarität. Meist tritt die Erkrankung erst ab dem neunten Lebensjahr auf, betonte Dr. Barbara Kunz aus Hamburg. Als Pathomechanismus wird ein Autoimmunprozess mit autoreaktiven TZellen, Autoantikörpern gegen Melanozytenantigene oder auch oxidativen Schäden vermutet. In der Histologie fehlen die Melanozyten beim betroffenen Hautareal, im Woodlicht („Schwarzlicht“) erscheinen diese Bereiche leuchtend weiß. Neben der Vitiligo vulgaris mit typisch symmetrischem

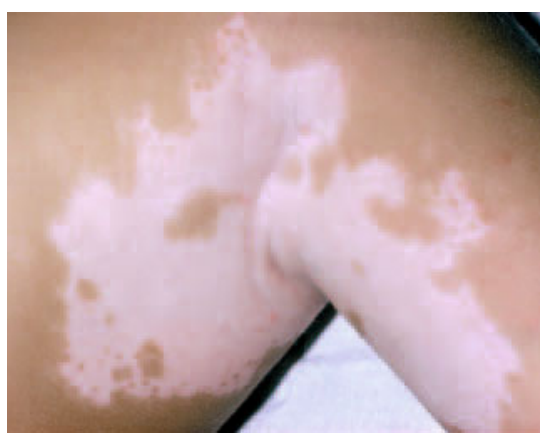

Zwei Fälle von Vitiligo
Zehen sowie rasch wachsende Hämangiome. Wichtig sei eine genaue Ausmessung und Dokumentation der befallenen Bereiche inklusive des Wachstumsverlaufs. Tiefere Anteile sollten per Ultraschall beurteilt werden - dies ermöglicht genaue Kenntnis über die Ausdehnung des Tumors und über die Durchblutung als Hinweis auf dessen Aktivität.

Bause H. Kryotherapie lokalisierter

Hämangiome geschützt werden.
Zur Kryotherapie stehen zurzeit zwei Geräte zur Verfügung: „Cryocare“ der Firma gbo und "Kryoderm“ von Ergomed. Mit diesen Geräten ist eine Behandlung mit $-32{ }^{\circ} \mathrm{C}$ möglich. Gegenüber der früheren Methode mit flüssigem Stickstoff $\left(-196^{\circ} \mathrm{C}\right)$, bei der die Gefahr einer ausgedehnten Nekrose besteht, wird die Einwirkzeit auf 20 Sekunden verdoppelt. Hilfreich zur Behandlung ist das Auftragen anästhesierender Salben, wie z.B. Emla ${ }^{\circledR}$, auf die betreffenden Hautstellen eine halbe bis eine ganze Stunde vor der Behandlung. Mithilfe der Kryotherapie lassen sich Hämangiome von 2-3 mm Größe gut behandeln, mit kräftigem Druck sogar bis zu $5 \mathrm{~mm}$, so Bause. Für tiefer reichende Gefäßfehlbildungen ist die Lasertherapie besser geeignet. Nach der Kryotherapie empfiehlt sich das Auftragen von antiseptischen Lösungen oder Salben. Die neugebildete Haut sollte vor Sonneneinwirkung

af

Befall (beispielsweise Streckseiten der Knie, Unterschenkel, Füße, Ellbogen und Handrücken), kann die Erkrankung auch lokal,

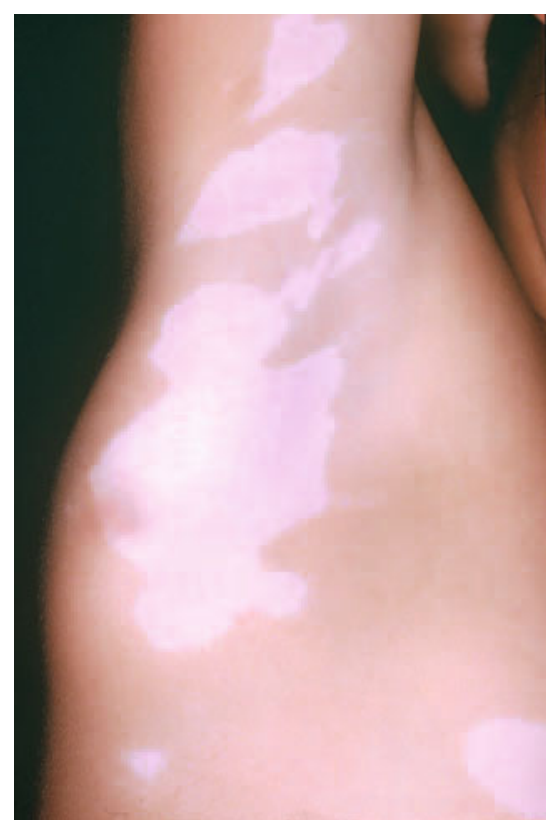

entlang einem Hautsegment oder generalisiert auftreten.

Die Patienten sollten regelmäßig untersucht werden. Die Prognose ist bei segmentalem Befall meist besser als bei diffuser symmetrischer Ausbreitung. Bei hellem Hauttyp (Typ I und II) kann abgewartet werden; mitunter ist in bis zu 30\% der Fälle eine Spontanremission möglich. Bei kleineren Arealen kommt eine Behandlung mit modernen topischen Steroiden zweimal täglich für drei Wochen in Frage. Auch Calcineurininhibitoren (Pimecrolimus zweimal täglich für acht Wochen, Tacrolimus zweimal täglich für sechs Wochen) werden eingesetzt. Bei ausgedehntem Befund und dunklem Hauttyp (Hauttyp III und IV) ist eine Bestrahlung mit UVB-Licht möglich. Eine Kombinationstherapie aus topischem Psoralen und UVA-Licht, „Creme-PUVA“, stellt ebenfalls eine Therapieoption dar. Hier sollte jedoch auf das erhöhte Hautkrebsrisiko geachtet werden.

Kunz B. Spektrum der hypopigmentierten Hautververänderungen 